\title{
Genome sequences of Bacteria and Archaea published outside of Standards in Genomic Sciences, June - September 2011
}

\author{
Oranmiyan W. Nelson ${ }^{1}$ and George M. Garrity ${ }^{1}$ \\ ${ }^{1}$ Editorial Office, Standards in Genomic Sciences and Department of Microbiology, \\ Michigan State University, East Lansing, MI, USA
}

The purpose of this table is to provide the community with a citable record of publications of ongoing genome sequencing projects that have led to a publication in the scientific literature. While our goal is to make the list complete, there is no guarantee that we may have omitted one or more publications appearing in this time frame. Readers and authors who wish to have publications added to this subsequent versions of this list are invited to provide the bibliometric data for such references to the SIGS editorial office.

\section{Phylum Crenarchaeota}

\section{Phylum Euryarchaeota}

Pyrococcus yayanosii CH1, sequence accession CP002779 [1]

Methanocella paludicola, sequence accession AP011532 [2]

Halorhabdus tiamatea, sequence accession AFNT00000000 [3]

Thermococcus sp. Strain 4557, sequence accession CP002920 [4]

\section{Phylum Chloroflexi}

\section{Phylum Proteobacteria}

Ralstonia solanacearum strain Po82, sequence accession CP002819 (chromosome) and CP002820 (megaplasmid) [5

Desulfovibrio alaskensis G20, sequence accession CP000112 [6]

Methylophaga aminisulfidivorans MPT, sequence accession AFIG00000000 [7]

Acinetobacter sp. P8-3-8, sequence accession AFIE00000000 [8]

Sphingomonas strain KC8, sequence accession AFMP01000000 [9]

Brucella pinnipedialis B2/94, sequence accession CP002078 and CP002079 [10]

Salmonella enterica Serovar Typhimurium UK-1, sequence accession CP002614

(chromosome), CP002615 (plasmid) [11]

Bordetella pertussis CS, sequence accession CP002695 [12]

Alteromonas sp. Strain SN2, sequence accession CP002339 [13]

Escherichia coli 0104:H4, sequence accession AFOB00000000 (LB226692) and

AFPS00000000 (01-09591) [14]

Acidithiobacillus caldus, sequence accession CP002573 (Chromosome), CP002574

(pLAtcm), CP002575 (pLAtc1), CP002576 (pLAtc2), CP002577 (pLAtc3) [15] 
Cupriavidus necator N-1, sequence accession CP002877 (chromosome 1), CP002878 (chromosome 2), CP002879 (pBB1), and CP002880 (pBB2) [16]

Oligotropha carboxidovorans OM4, sequence accession CP002821 (OM4 chromosome), CP002822 (pHCG3b), CP002823 (p0C167B) [17]

Oligotropha carboxidovorans OM5, sequence accession CP002826 (OM5 chromosome), CP002827 (pHCG3), and CP002828 (p0C167) [17]

Pantoea ananatis LMG20103, sequence accession CP001875 [18]

Helicobacter bizzozeronii strain CIII-1, sequence accession FR871757 (chromosome) and FR871758 (HBZ-1) [19]

Vibrio anguillarum 775, sequence accession CP002284 to CP002285 [20]

Zymomonas mobilis subsp. pomaceae, sequence accession CP002865 (chromosome), CP002866 (p29192_1), CP002867 (p29192_2) [21]

Agrobacterium sp. strain ATCC 31749, sequence accession AECL01000000 [22]

Xanthomonas spp. strain Xrc, sequence accesssion CP002789 [23]

Xanthomonas spp. strain Xoc, sequence accesssion AAQN00000000 [23]

Glaciecola sp. Strain 4H-3-7+YE-5, sequence accession CP002526 (chromosome) and CP002527 (plasmid) [24]

Escherichia coli Strain HM605, sequence accession CADZ01000001 through CADZ01000154 [25]

Salinisphaera shabanensis, sequence accession AFNV00000000 [26]

Methyloversatilis universalis FAM5T, sequence accession AFHG00000000 [27]

Alicycliphilus denitrificans Strain BC, sequence accession CP002449 (chromosome), CP002450 (megaplasmid), CP002451 (plasmid) [28].

Alicycliphilus denitrificans $\mathrm{K} 601^{\mathrm{T}}$, sequence accession CP002657 (chromosome) and CP002658 (plasmid) [28]

Oligotropha carboxidovorans Strain OM4, sequence accession CP002821 (chromosome), CP002822 (pHCG3b), CP002823 (p0C167B) [29]

Oligotropha carboxidovorans Strain OM5, sequence accession CP002826 (chromosome), CP002827 (pHCG3), and CP002828 (p0C167) [29]

Bradyrhizobiaceae strain SG-6C, sequence accession AF0F01000000 [30]

Hyphomicrobium sp. Strain MC1, sequence accession FQ859181 [31]

Shewanella sp. Strain HN-41, sequence accession AFOZ01000000 [32]

Myxococcus fulvus HW-1, sequence accession CP002830 [33]

Nitrosomonas sp. Strain AL212, sequence accession NC_015222 (chromosome),

NC_015223 pNAL21201), NC_015221 (pNAL21202) [34]

Ruegeria sp. Strain KLH11, sequence accession ACCW00000000 [35]

Acidovorax avenae subsp. avenae RS-1, sequence accession AFPT01000000 [36]

Escherichia coli (ExPEC), sequence accession AFAT00000000 [37] 
Vibrio mimicus SX-4, sequence accession AD0001000000 [38]

Agrobacterium tumefaciens Strain F2, sequence accession AFSD00000000 [39]

Pasteurella multocida subsp. gallicida AFRR01000001 to AFRR01000489 [40]

Pseudomonas aeruginosa 138244, sequence accession AEVV00000000 [41]

Pseudomonas aeruginosa 152504, sequence accession AEVW00000000 [41]

Campylobacter jejuni strain 305, sequence accession ADHL00000000 [42]

Campylobacter jejuni strain DFVF1099, sequence accession ADHK00000000 [42]

Xanthomonas campestris pv. raphani strain 756C, sequence accession CP002789 [43]

Xanthomonas campestris pv. raphani strain BLS256, sequence accession AAQN01000001 [43]

Rickettsia heilongjiangensis, sequence accession CP002912 [44]

Acidiphilium sp. Strain PM (DSM 24941), sequence accession AFPR00000000 [45]

Pseudomonas putida Strain S16, sequence accession CP002870 [46]

Acinetobacter lwoffii, sequence accession AFQY01000000 [47]

\section{Phylum Firmicutes}

Caldalkalibacillus thermarum strain TA2.A1, sequence accession AFCE00000000 [48]

Listeria monocytogenes Scott A, sequence accession AFGI00000000 [49]

Lactococcus garvieae 8831, sequence accession AFCD00000000 [50]

Natranaerobius thermophilus JW/NM-WN-LF, sequence accession CP001034 (chromosome), CP001035 (plasmid) [51]

Melissococcus plutonius ATCC 35311, sequence accession AP012200 (chromosome) and AP012201 (plasmid) [52]

Lactobacillus buchneri NRRL B-30929, sequence accession CP002652 (chromosome), CP002653 (plasmid pLBU01), CP002654 (plasmid pLBU02), and CP002655 (plasmid pLBU03) [53]

Lactobacillus kefiranofaciens ZW3, sequence accession CP002764 (chromosome), CP002765 (plasmid), and CP002766 (plasmid) [54]

Bacillus megaterium strain QM B1551, sequence accession CP001983 (chromosome), CP001984 to CP001990 (plasmids pBM100 through pBM700) [55]

Bacillus megaterium strain DSM319, sequence accession CP001982 (chromosome) [55]

Listeria monocytogenes serovar 4a strain M7, sequence accession CP002816 [56]

Bacillus coagulans 2-6, sequence accession CP002472 [57]

Streptococcus salivarius strain CCHSS3, sequence accession FR873481 [58]

Paenibacillus elgii B69, sequence accession AFHW01000000 [59]

Lactobacillus pentosus MP-10, sequence accession FR871759 through FR871848 [60] 
Leuconostoc pseudomesenteroides KCTC 3652, sequence accession AEOQ00000001 through AEOQ00001160 [61]

Lactobacillus mali KCTC 3596, sequence accession BACP01000001 through BACP01000122 [62]

Paenibacillus polymyxa Type Strain ATCC $842^{\mathrm{T}}$, sequence accession AFOX01000000 [63]

Streptococcus salivarius strain JIM8777, sequence accssion FR873482 [64]

Lactobacillus cypricasei KCTC 13900, sequence accession BACS01000001 to BACS01000487 [65]

Lactobacillus zeae KCTC 3804, sequence accession BACQ01000001 to BACQ101000113 [66]

Listeria monocytogenes Serovar 4a Strain M7, sequence accession CP002816 [67]

Lactobacillus salivarius GJ-24, sequence accession AFOI00000000 [68]

Lactobacillus johnsonii PF01, sequence accession AFQJ01000000 [69]

Clostridium acetobutylicum DSM 1731, sequence accession CP002660 through

CP002662 [70]

Lactobacillus suebicus KCTC 3549, sequence accession BAC001000000 [71]

Brevibacillus laterosporus LMG 15441, sequence accession AFRV00000000 [72]

Lactobacillus salivarius NIAS840, sequence accession AFMN00000000 [73]

Bifidobacterium animalis subsp. lactis CNCM I-2494, sequence accession CP002915 [74]

Megasphaera elsdenii, sequence accession HE576794 [75]

Lactobacillus versmoldensis KCTC 3814, sequence accession BACR01000001 to BACR01000102 [76]

Lactobacillus pentosus IG1, sequence accession FR874848 to FR874860 [77]

Alicyclobacillus acidocaldarius Strain Tc-4-1, sequence accession CP002902 [78]

Streptococcus thermophilus Strain JIM8232, sequence accession FR875178 [79]

Streptococcus equi subsp. zooepidemicus Strain ATCC 35246, sequence accession CP002904 [80]

Bacillus amyloliquefaciens XH7, sequence accession CP002927 [81]

Leuconostoc kimchii Strain C2, sequence accession CP002898 [82]

Lactobacillus malefermentans KCTC 3548, sequence accession BACN01000001 to BACN01000172 [83]

Weissella koreensis KACC 15510, sequence accession CP002900 [84]

\section{Phylum Tenericutes}

Mycoplasma bovis Strain Hubei-1, sequence accession CP002513 [85]

Mycoplasma fermentans Strain M64, sequence accession NC_014921 [86] 
Haloplasma contractile, sequence accession AFNU00000000 [87]

Mycoplasma ovipneumoniae Strain SC01, sequence accession AFH001000000 [88]

\section{Phylum Actinobacteria}

Kocuria rhizophila P7-4, sequence accession AFID00000000 [89]

Streptomyces S4, sequence accession CADY01000000 [90]

Corynebacterium nuruki S6-4T, sequence accession AFIZ00000000 [91]

Propionibacterium humerusii, sequence accession AFAM00000000.1 [92]

Strain JDM601, sequence accession CP002329 [93]

Streptomyces sp. strain Tü6071, sequence accession AFHJ01000000 [94]

Bifidobacterium breve UCC2003, sequence accession CP000303 [95]

Propionibacterium acnes, sequence accession CP002815 [96]

Amycolicicoccus subflavus DQS3-9A1T, sequence accession CP002786 (chromosome), CP002787 (plasmid pAS9A-1), and CP002788 (plasmid pAS9A-2). [97]

Gordonia neofelifaecis NRRL B-59395, sequence accession AEUD01000000 [98]

Pseudonocardia dioxanivorans strain CB1190, sequence accession NC_015312-4 and CP002595-7 [99]

Bifidobacterium longum subsp. longum KACC 91563, sequence accession CP002794 to CP002796 [100]

Streptomyces cattleya NRRL 8057, sequence accession FQ859185 (chromosome) and FQ859184 (megaplasmid) [101]

Rhodococcus sp. Strain R04, sequence accession AFAQ01000000 [102]

Mycobacterium bovis BCG Moreau, sequence accession [103]

Saccharopolyspora spinosa NRRL 18395, sequence accession [104]

Mycobacterium tuberculosis CCDC5079, sequence accession [105]

Mycobacterium tuberculosis CCDC5180, sequence accession [105]

Amycolatopsis mediterranei S699, sequence accession CP002896 [106]

Nesterenkonia sp. Strain F, sequence accession AFRW01000000 [107]

Streptomyces xinghaiensis NRRL B24674T, sequence accession AFRP01000000 [108]

\section{Phylum Chlamydiae}

Chlamydophila abortus variant strain LLG, sequence accession AFHM01000000 [109]

Chlamydia psittaci 6BC, sequence accession CP002586 (chromosome), CP002587 (plasmid) [110]

Chlamydia psittaci Cal10, sequence accession AEZD00000000 (draft chromosome and plasmid) [110]

Chlamydia trachomatis, sequence accession CP002024 [111]

\section{Phylum Spirochaetes}


Spirochaeta thermophila DSM 6192, sequence accession CP001698 [112]

Brachyspira intermedia, sequence accession CP002874 (chromosome) and CP002875 (plasmid) [113]

\section{Phylum Fibrobacteres}

\section{Phylum Bacteroidetes}

Porphyromonas gingivalis TDC60, sequence accession AP012203 [114]

Krokinobacter sp. strain 4H-3-7-5, sequence accession CP002528 [115]

Lacinutrix sp. strain 5H-3-7-4, sequence accession CP002825 [115]

Bacterium HQM9, sequence accession AFPB00000000 [116]

Anaerophaga sp. Strain HS1, sequence accession AFSL00000000 [117]

Capnocytophaga canimorsus Strain 5, sequence accession CP002113 [118]

Mesoflavibacter zeaxanthinifaciens strain S86, sequence accession AFOE00000000 [119]

\section{Phylum Verrucomicrobia}

\section{Phylum Lentisphaerae}

Phylum Thermotogae

Kosmotoga olearia Strain TBF 19.5.1, sequence accession CP001634 [120]

\section{Domain Archaea}

"Candidatus Nitrosoarchaeum koreensis" MY1, sequence accession AFPU00000000 [121]

\section{Non-Bacterial genomes}

Cucumis sativus L., North-European Cucumber, sequence accession FI132140-FI136208, GS765762-GS766880, GS815969-GS874855 [122]

Ricinus communis Castor bean organelle genome, sequence accession JF937588(chloroplast), HQ874649 (mitochondria) [123]

Stretch Lagoon Orbivirus Umatilla, sequence accession HQ842619 through HQ842628 [124]

Gadus morhua Atlantic cod, sequence accession CAEA01000001 through CAEA01554869 [125]

Solanum tuberosum L. Potato, sequence accession GS025503 through GS026177 [126]

ФCA82, sequence accession HQ264138 [127]

Paramecium caudatumreveals mitochondria, sequence accession NC001324 [128]

bacteriophage IME08, sequence accession NC_014260 [129]

virus (ILTV), sequence accession HQ_630064 [130]

Macropus eugenii Australian kangaroo, sequence accession ABQ0000000000 [131]

Aichi virus, sequence accession FJ890523 [132]

"Candidatus Tremblaya princeps" Strain PCVAL, sequence accession CP002918 [133] 


\section{References}

1. Jun X, Lupeng L, Minjuan X, Oger P, Fengping W, Jebbar M, Xiang X. Complete Genome Sequence of the Obligate Piezophilic Hyperthermophilic Archaeon Pyrococcus yayanosii CH1. I Bacteriol 2011; 193:4297-4298.

PubMed doi:10.1128/JB.05345-11

2. Sakai S, Takaki Y, Shimamura S, Sekine M, Tajima T, Kosugi H, Ichikawa N, Tasumi E, Hiraki A, Shimizu A, et al. Genome Sequence of a Mesophilic Hydrogenotrophic Methanogen Methanocella paludicola, the First Cultivated Representative of the Order Methanocellales. [date unknown]. PLOS ONE 2011; 6:e22898. PubMed doi:10.1371/journal.pone.0022898

3. Antunes A, Alam I, Bajic VB, StingI U. Genome Sequence of Halorhabdus tiamatea, the First Archaeon Isolated from a Deep-Sea Anoxic Brine Lake. J Bacteriol 2011; 193:4553-4554. PubMed doi:10.1128/JB.05462-11

4. Wang X, Gao Z, Xu X, Ruan L. Complete Genome Sequence of Thermococcus sp. Strain 4557, a Hyperthermophilic Archaeon Isolated from a Deep-Sea Hydrothermal Vent Area. I Bacteriol 2011; 193:5544-5545.

PubMed doi:10.1128/JB.05851-11

5. Xu J, Zheng HJ, Liu L, Pan ZC, Prior P, Tang B, Xu JS, Zhang H, Tian Q, Zhang LQ, et al. The complete genome sequence of plant pathogen Ralstonia solanacearum strain Po82. J Bacteriol 2011; 193:4261-4262. PubMed doi:10.1128//B.05384$\underline{11}$

6. Hauser LJ, Land ML, Brown SD, Larimer F, Keller KL, Rapp-Giles BJ, Price MN, Lin M, Bruce DC, Detter JC, et al. The Complete Genome Sequence and Updated Annotation of Desulfovibrio alaskensis G20. J Bacteriol 2011; 193. PubMed doi:10.1128/JB.05400-11

7. Han GH, Kim W, Chun J, Kim SW. Draft genome sequence of Methylophaga aminisulfidivorans MPT . J Bacteriol 2011;

8. Kim YO, Kim WJ, Choi SH, Kim DS, Kim DW, Lee JS, Kong HJ, Nam BH, Kim BS, Lee SJ, et al. Genome Sequence of Acinetobacter sp. P8-3-8, isolated from Fistularia commersonii in Vietnam . J Bacteriol 2011;

9. Hu A, He J, Chu KH, Yu CP. Genome Sequence of a 17 -Estradiol-Utilizing Bacterium Sphingomonas strain KC8 . J Bacteriol 2011;

10. Audic S, Lescot M, Claverie JM, Cloeckaert A, Zygmunt M. The genome sequence of Brucella pinnipedialis B2/94 sheds light on the evolutio- nary history of the genus Brucella. BMC Evol Biol $2011 ; 11: 200$. PubMed doi:10.1186/1471-2148$\underline{11-200}$

11. Luo Y, Kong Q, Yang J, Golden G, Wanda SY, Jensen RV, Ernst PB, Curtiss R. Complete Genome Sequence of the Universal Killer Salmonella enterica Serovar Typhimurium UK-1 (ATCC 68169). J Bacteriol 2011; 193:4035-4036. PubMed doi:10.1128//B.05224-11

12. Zhang S, Xu Y, Zhou Z, Wang S, Yang R, Wang J, Wang L. Complete Genome Sequence of Bordetella pertussis CS, a Chinese Pertussis Vaccine Strain. J Bacteriol 2011; 193:4017-4018. PubMed doi:10.1128/JB.05184-11

13. Jin $\mathrm{HM}$, Jeong $\mathrm{H}$, Moon EJ, Math RK, Lee $\mathrm{K}$, Kim HJ, Jeon CO, Oh TK, Kim JF. Complete Genome Sequence of the Polycyclic Aromatic Hydrocarbon-Degrading Bacterium Alteromonas sp. Strain SN2. I Bacteriol 2011; 193:4292-4293.

PubMed doi:10.1128//B.05252-11

14. Mellmann A, Harmsen D, Cummings C, Zentz E, Leopold S, Rico A, Prior K, Szczepanowski R, Ji Y, Zhang W, et al. Prospective Genomic Characterization of the German Enterohemorrhagic Escherichia coli O104:H4 Outbreak by Rapid Next Generation Sequencing Technology. PLOS ONE 2011; 6:e22751. PubMed doi:10.1371/journal.pone.0022751

15. You XY, Guo X, Zheng HJ, Zhang MJ, Liu LJ, Zhu YQ, Zhu B, Wang SY, Zhao GP, Poetsch A, et al. Unraveling the Acidithiobacillus caldus complete genome and its central metabolisms for carbon assimilation. J Genet Genomics 2011; 38:243252. PubMed doi:10.1016/j.jgg.2011.04.006

16. Poehlein A, Kusian B, Friedrich B, Daniel R, Bowien $B$. Complete genome sequence of the type strain Cupriavidus necator N-1 . J Bacteriol 2011;

17. Volland S, Rachinger M, Strittmatter A, Daniel R, Gottschalk G, Meyer O. Complete genome sequences of the chemolithoautotrophic strains Oligotropha carboxidovorans OM4 and OM5. J Bacteriol 2011;

18. De Maayer P, Chan WY, Venter SN, Toth IK, Birch PRJ, Joubert F, Coutinho TA. Genome Sequence of Pantoea ananatis LMG20103, the Causative Agent of Eucalyptus Blight and Dieback. J Bacteriol 2010; 192:2936-2937.

PubMed doi:10.1128/JB.00060-10

19. Schott T, Rossi M, Hanninen ML. Genome sequence of Helicobacter bizzozeronii strain CIII-1, 
an isolate from human gastric mucosa . J Bacteriol 2011;

20. Naka H, Dias GM, Thompson CC, Dubay C, Thompson FL, Crosa JH. Complete Genome Sequence of the Marine Fish Pathogen Vibrio anguillarum Harboring the pJM1 Virulence Plasmid and Genomic Comparison with Other Virulent Strains of $V$. anguillarum and $V$. ordalii. Infect Immun 2011; 79:2889-2900.

PubMed doi:10.1128/IAI.05138-11

21. Kouvelis VN, Davenport KW, Brettin TS, Bruce D, Detter C, Han C, Nolan M, Tapia R, Damoulaki A, Kyrpides NC, et al. Genome sequence of the ethanol-producing Zymomonas mobilis subsp. pomaceae lectotype ATCC 29192 . J Bacteriol 2011;

22. Ruffing AM, Castro-Melchor M, Hu WS, Chen RR. Genome Sequence of the Curdlan-Producing Agrobacterium sp. Strain ATCC 31749. J Bacteriol 2011; 193:4294-4295.

PubMed doi:10.1128/JB.05302-11

23. Bogdanove AJ, Koebnik R, Lu H, Furutani A, Angiuoli SV, Patil PB, Van Sluys MA, Ryan RP, Meyer DF, Han SW, et al. Two new complete genome sequences offer insight into host and tissue specificity of plant pathogenic Xanthomonas spp. . J Bacteriol 2011;

24. Klippel B, Lochner A, Bruce DC, Walston Davenport K, Detter C, Goodwin LA, Han J, Han S, Land ML, Mikhailova N, et al. Complete Genome Sequence of the Marine Cellulose- and XylanDegrading Bacterium Glaciecola sp. Strain 4H-37+YE-5. J Bacteriol 2011; 193:4547-4548. PubMed doi:10.1128//B.05468-11

25. Clarke DJ, Chaudhuri RR, Martin HM, Campbell BJ, Rhodes JM, Constantinidou C, Pallen MJ, Loman NJ, Cunningham AF, Browning DF, et al. Complete Genome Sequence of the Crohn's Disease-Associated Adherent-Invasive Escherichia coli Strain HM605. J Bacteriol 2011; 193:4540. PubMed doi:10.1128/JB.05374-11

26. Antunes A, Alam I, Bajic VB, Stingl U. Genome Sequence of Salinisphaera shabanensis, a Gammaproteobacterium from the Harsh, Variable Environment of the Brine-Seawater Interface of the Shaban Deep in the Red Sea. J Bacteriol 2011; 193:4555-4556. PubMed doi:10.1128/JB.0545911

27. Kittichotirat W, Good NM, Hall R, Bringel F, Lajus A, Medigue C, Smalley NE, Beck D, Bumgarner R, Vuilleumier $S$, et al. Genome Sequence of Methyloversatilis universalis FAM5T, a Methy- lotrophic Representative of the Order Rhodocyclales. J Bacteriol 2011; 193:4541-4542.

PubMed doi:10.1128/JB.05331-11

28. Oosterkamp MJ, Veuskens T, Plugge CM, Langenhoff AAM, Gerritse J, van Berkel WJH, Pieper $\mathrm{DH}$, Junca $\mathrm{H}$, Goodwin LA, Daligault HE, et al. Genome Sequences of Alicycliphilus denitrificans Strains BC and K601 ${ }^{\top}$. J Bacteriol 2011;

193:5028-5029. PubMed doi:10.1128/JB.0036511

29. Volland S, Rachinger M, Strittmatter A, Daniel R, Gottschalk G, Meyer O. Complete Genome Sequences of the Chemolithoautotrophic Oligotropha carboxidovorans Strains OM4 and OM5. J

Bacteriol 2011; 193:5043. PubMed doi:10.1128/]B.05619-11

30. Pearce SL, Pandey R, Dorrian SJ, Russell RJ, Oakeshott JG, Pandey G. Genome Sequence of the Newly Isolated Chemolithoautotrophic Bradyrhizobiaceae Strain SG-6C. J Bacteriol 2011; 193:5057. PubMed doi:10.1128/JB.05647-11

31. Vuilleumier $S$, Nadalig T, Farhan UI Haque $M$, Magdelenat G, Lajus A, Roselli S, Muller EEL, Gruffaz C, Barbe V, Medigue C, et al. Complete Genome Sequence of the ChloromethaneDegrading Hyphomicrobium sp. Strain MC1. I Bacteriol 2011; 193:5035-5036. PubMed doi:10.1128/JB.05627-11

32. Kim DH, Jiang S, Lee JH, Cho YJ, Chun J, Choi SH, Park HS, Hur HG. Draft Genome Sequence of Shewanella sp. Strain HN-41, Which Produces Arsenic-Sulfide Nanotubes. J Bacteriol 2011; 193:5039-5040. PubMed doi:10.1128/JB.0557811

33. Li ZF, Li X, Liu H, Liu X, Han K, Wu ZH, Hu W. Li F-fei, Li Y-Z: Genome Sequence of the Halotolerant Marine Bacterium Myxococcus fulvus HW1. J Bacteriol 2011; 193:5015-5016. PubMed doi:10.1128//B.05516-11

34. Yuichi S, Norton JM, Bollmann A, Klotz MG, Stein LY, Laanbroek HJ, Arp DJ, Goodwin LA, Chertkov O, Held B, et al. Genome Sequence of Nitrosomonas sp. Strain AL212, an AmmoniaOxidizing Bacterium Sensitive to High Levels of Ammonia. J Bacteriol 2011; 193:5047-5048. PubMed doi:10.1128/JB.05521-11

35. Zan J, Fricke WF, Fuqua C, Ravel J, Hill RT. Genome Sequence of Ruegeria sp. Strain KLH11, an $\mathrm{N}$-Acylhomoserine Lactone-Producing Bacterium Isolated from the Marine Sponge Mycale laxissima. J Bacteriol 2011; 193:5011-5012.

PubMed doi:10.1128/JB.05556-11 
36. Xie GL, Zhang GQ, Liu H, Lou MM, Tian WX, Li B, Zhou XP, Zhu B, Jin GL. Genome Sequence of the Rice-Pathogenic Bacterium Acidovorax avenae subsp. avenae RS-1. J Bacteriol 2011;

193:5013-5014. PubMed doi:10.1128/JB.0559411

37. Tan C, Xu Z, Zheng H, Liu W, Tang X, Shou J, Wu B, Wang S, Zhao GP, Chen H. Genome Sequence of a Porcine Extraintestinal Pathogenic Escherichia coli Strain. J Bacteriol 2011; 193:5038. PubMed doi:10.1128//B.05551-11

38. Wang D, Wang H, Zhou Y, Zhang Q, Zhang F, Du P, Wang S, Chen C, Kan B. Genome Sequencing Reveals Unique Mutations in Characteristic Metabolic Pathways and the Transfer of Virulence Genes between V. mimicus and V. cholerae. PLOS ONE 2011; 6:e21299.

PubMed doi:10.1371/journal.pone.0021299

39. Li A, Geng J, Cui D, Shu C, Zhang S, Yang J, Xing J, Wang J, Ma F, Hu S. Genome Sequence of Agrobacterium tumefaciens Strain F2, a Bioflocculant-Producing Bacterium. J Bacteriol 2011; 193:5531. PubMed doi:10.1128/JB.05690-11

40. Ahir VB, Roy A, Jhala MK, Bhanderi BB, Mathakiya RA, Bhatt VD, Padiya KB, Jakhesara SJ, Koringa PG, Joshi CG. Genome Sequence of Pasteurella multocida subsp. gallicida Anand1_poultry. J Bacteriol 2011; 193:5604.

PubMed doi:10.1128/JB.05706-11

41. Soares-Castro P, Marques D, Demyanchuk S, Faustino A, Santos PM. Draft Genome Sequences of Two Pseudomonas aeruginosa Clinical Isolates with Different Antibiotic Susceptibilities. J Bacteriol 2011; 193:5573. PubMed doi:10.1128//B.05446-11

42. Takamiya M, Ozen A, Rasmussen M, Alter T, Gilbert T, Ussery DW, Knochel S. Genome Sequences of Two Stress-Tolerant Campylobacter jejuni Poultry Strains, 305 and DFVF1099. J Bacteriol 2011; 193:5546-5547.

PubMed doi:10.1128/JB.05753-11

43. Bogdanove AJ, Koebnik R, Lu H, Furutani A, Angiuoli SV, Patil PB, Van Sluys MA, Ryan RP, Meyer DF, Han SW, et al. Two New Complete Genome Sequences Offer Insight into Host and Tissue Specificity of Plant Pathogenic Xanthomonas spp. J Bacteriol 2011; 193:5450-5464. $\underline{\text { PubMed doi:10.1128/JB.05262-11 }}$

44. Duan C, Tong Y, Huang Y, Wang X, Xiong X, Wen B. Complete Genome Sequence of Rickettsia heilongjiangensis, an Emerging Tick-Transmitted
Human Pathogen. J Bacteriol 2011; 193:5564-

5565. PubMed doi:10.1128//B.05852-11

45. San Martin-Uriz P, Gomez MJ, Arcas A, Bargiela R, Amils R. Draft Genome Sequence of the Electricigen Acidiphilium sp. Strain PM (DSM 24941). J Bacteriol 2011; 193:5585-5586. PubMed doi:10.1128//B.05386-11

46. Yu H, Tang H, Wang L, Yao Y, Wu G, Xu P. Complete Genome Sequence of the NicotineDegrading Pseudomonas putida Strain S16. J Bacteriol 2011; 193:5541-5542.

PubMed doi:10.1128/JB.05663-11

47. Hu Y, Zhang W, Liang H, Liu L, Peng G, Pan Y, Yang X, Zheng B, Gao GF, Zhu B, et al. WholeGenome Sequence of a Multidrug-Resistant Clinical Isolate of Acinetobacter Iwoffii. J Bacteriol 2011; 193:5549-5550. PubMed doi:10.1128//B.05617-11

48. Kalamorz F, Keis S, McMillan DGG, Olsson K, Stanton J-A, Stockwell P, Black MA, Klingeman DM, Land ML, Han CS, et al.: Draft Genome Sequence of Thermoalkaliphilic Caldalkalibacillus thermarum strain TA2.A1. J. Bacteriol. 2011,

49. Briers Y, Klumpp J, Schuppler M, Loessner MJ. Genome sequence of Listeria monocytogenes Scott A, a clinical isolate from a foodborne listeriosis outbreak . J Bacteriol 2011;

50. Aguado-Urda M, Lopez-Campos GH, Gibello A, Cutuli MT, Lopez-Alonso V, Fernandez-

Garayzabal JF, Blanco MM. Genome sequence of Lactococcus garvieae 8831, isolated from rainbow trout lactococcosis outbreaks in Spain . J Bacteriol 2011;

51. Zhao B, Mesbah NM, Dalin E, Goodwin L, Nolan M, Pitluck S, Chertkov O, Brettin TS, Han J, Larimer FW, et al. Complete Genome Sequence of the Anaerobic, Halophilic Alkalithermophile Natranaerobius thermophilus JW/NM-WN-LF. J Bacteriol 2011; 193:4023-4024.

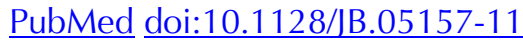

52. Okumura K, Arai R, Okura M, Kirikae T, Takamatsu D, Osaki M, Miyoshi-Akiyama T. Complete Genome Sequence of Melissococcus plutonius ATCC 35311. J Bacteriol 2011; 193:4029-4030. PubMed doi:10.1128/JB.05151-11

53. Liu S, Leathers TD, Copeland A, Chertkov O, Goodwin L, Mills DA. Complete Genome Sequence of Lactobacillus buchneri NRRL B-30929, a Novel Strain from a Commercial Ethanol Plant. J Bacteriol 2011; 193:4019-4020. PubMed doi:10.1128//B.05180-11 
54. Wang Y, Wang J, Ahmed Z, Bai X, Wang J. Complete Genome Sequence of Lactobacillus kefiranofaciens ZW3. J Bacteriol 2011; 193:4280-4281. PubMed doi:10.1128/JB.05306-11

55. Eppinger $M$, Bunk $B$, Johns MA, Edirisinghe JN, Kutumbaka KK, Koenig SSK, Huot Creasy H, Rosovitz MJ, Riley DR, Daugherty S, et al. Genome Sequences of the Biotechnologically Important Bacillus megaterium Strains QM B1551 and DSM319. J Bacteriol 2011; 193:4199-4213. PubMed doi:10.1128//B.00449-11

56. Chen J, Xia Y, Cheng C, Fang C, Shan Y, Jin G, Fang W. Genome sequence of a non-pathogenic Listeria monocytogenes serovar 4a strain M7. J Bacteriol 2011;

57. Su F, Yu B, Sun J, Ou HY, Zhao B, Wang L, Qin J, Tang $H$, Tao F, Jarek $M$, et al. Genome Sequence of Thermophilic Strain Bacillus coagulans 2-6, an Efficient Producer of High Optical Purity L-Lactic Acid. J Bacteriol 2011;

58. Delorme C, Guedon E, Pons N, Cruaud C, Couloux A, Loux V, Chiapello H, Poyart C, Gautier C, Sanchez N, et al. Complete Genome Sequence of the clinical Streptococcus salivarius strain CCHSS3 . J Bacteriol 2011; 10.1128/JB.05416-11.

59. Ding R, Li Y, Qian C, Wu X. Draft Genome Sequence of Paenibacillus elgii B69, a Strain with Broad Antimicrobial Activity. I Bacteriol 2011; 193:4537. PubMed doi:10.1128//B.00406-11

60. Abriouel H, Benomar N, Perez Pulido R, Canamero MM, Galvez A. Annotated Genome Sequence of Lactobacillus pentosus MP-10, Which Has Probiotic Potential, from Naturally Fermented Alorena Green Table Olives. I Bacteriol 2011; 193:4559-4560. PubMed doi:10.1128//B.0517111

61. Kim DW, Choi SH, Kang A, Nam SH, Kim RN, Kim A, Kim DS, Park HS. Genome Sequence of Leuconostoc pseudomesenteroides KCTC 3652. I Bacteriol 2011; 193:4299.

PubMed doi:10.1128//B.05433-11

62. Kim DW, Choi SH, Kang A, Nam SH, Kim DS, Kim RN, Kim A, Park HS. Draft Genome Sequence of Lactobacillus mali KCTC 3596. I Bacteriol 2011; 193:5037. PubMed doi:10.1128//B.05686-11

63. Jeong $\mathrm{H}$, Park SY, Chung WH, Kim SH, Kim N, Park SH, Kim JF. Draft Genome Sequence of the Paenibacillus polymyxa Type Strain (ATCC 842T), a Plant Growth-Promoting Bacterium. / Bacteriol 2011; 193:5026-5027. PubMed doi:10.1128//B.05447-11
64. Guédon E, Delorme C, Pons N, Cruaud C, Loux V, Couloux A, Gautier C, Sanchez N, Layec S, Galleron $\mathrm{N}$, et al. Complete Genome Sequence of the Commensal Streptococcus salivarius Strain JIM8777. I Bacteriol 2011; 193:5024-5025. PubMed doi:10.1128/JB.05390-11

65. Kim DS, Choi SH, Kim DW, Kim RN, Nam SH, Kang A, Kim A, Park HS. Genome Sequence of Lactobacillus cypricasei KCTC 13900. J Bacteriol 2011; 193:5053-5054.

PubMed doi:10.1128//B.05659-11

66. Kim DW, Choi SH, Kang A, Nam SH, Kim DS, Kim RN, Kim A, Park HS. Draft Genome Sequence of Lactobacillus zeae KCTC 3804. J Bacteriol 2011; 193:5023. PubMed doi:10.1128//B.05602-11

67. Chen J, Xia Y, Cheng C, Fang C, Shan Y, Jin G, Fang W. Genome Sequence of the Nonpathogenic Listeria monocytogenes Serovar 4a Strain M7. J Bacteriol 2011; 193:5019-5020. PubMed doi:10.1128/JB.05501-11

68. Cho YJ, Choi JK, Kim JH, Lim YS, Ham JS, Kang DK, Chun J, Paik HD, Kim GB. Genome Sequence of Lactobacillus salivarius GJ-24, a Probiotic Strain Isolated from Healthy Adult Intestine. J Bacteriol 2011; 193:5021-5022.

PubMed doi:10.1128//B.05616-11

69. Lee JH, Chae JP, Lee JY, Lim JS, Kim GB, Ham JS, Chun J, Kang DK. Genome Sequence of Lactobacillus johnsonii PF01, Isolated from Piglet Feces. J Bacteriol 2011; 193:5030-5031. PubMed doi:10.1128/JB.05640-11

70. Bao G, Wang R, Zhu Y, Dong H, Mao S, Zhang Y, Chen Z, Li Y, Ma Y. Complete Genome Sequence of Clostridium acetobutylicum DSM 1731, a Solvent-Producing Strain with Multireplicon Genome Architecture. J Bacteriol 2011; 193:5007-5008. PubMed doi:10.1128/JB.05596-11

71. Nam SH, Choi SH, Kang A, Kim DW, Kim RN, Kim DS, Kim A, Park HS. Genome Sequence of Lactobacillus suebicus KCTC 3549. I Bacteriol 2011; 193:5532-5533.

PubMed doi:10.1128/JB.05814-11

72. Djukic M, Poehlein A, Thurmer A, Daniel R. Genome Sequence of Brevibacillus laterosporus LMG 15441, a Pathogen of Invertebrates. J Bacteriol 2011; 193:5535-5536. PubMed doi:10.1128//B.05696-11

73. Ham JS, Kim HW, Seol KH, Jang A, Jeong SG, Oh $\mathrm{MH}$, Kim DH, Kang DK, Kim GB, Cha CJ. Genome Sequence of Lactobacillus salivarius NIAS840, Isolated from Chicken Intestine. / Bac- 
teriol 2011; 193:5551-5552.

PubMed doi:10.1128//B.05688-11

74. Chervaux C, Grimaldi C, Bolotin A, Quinquis B, Legrain-Raspaud S, van Hylckama Vlieg JET, Denariaz G, Smokvina T. Genome Sequence of the Probiotic Strain Bifidobacterium animalis subsp. lactis CNCM I-2494. J Bacteriol 2011; 193:55605561. PubMed doi:10.1128/JB.05716-11

75. Marx H, Graf AB, Tatto NE, Thallinger GG, Mattanovich D, Sauer M. Genome Sequence of the Ruminal Bacterium Megasphaera elsdenii. J Bacteriol 2011; 193:5578-5579.

PubMed doi:10.1128/]B.05861-11

76. Kim DS, Choi SH, Kim DW, Kim RN, Nam SH, Kang A, Kim A, Park HS. Genome Sequence of Lactobacillus versmoldensis KCTC 3814. I Bacteriol 2011; 193:5589-5590.

PubMed doi:10.1128/JB.05708-11

77. Maldonado-Barragàn A, Caballero-Guerrero B, Lucena-Padros H, Ruiz-Barba JL. Genome Sequence of Lactobacillus pentosus IG1, a Strain Isolated from Spanish-Style Green Olive Fermentations. I Bacteriol 2011; 193:5605.

PubMed doi:10.1128/JB.05736-11

78. Chen Y, He Y, Zhang B, Yang J, Li W, Dong Z, Hu S. Complete Genome Sequence of Alicyclobacillus acidocaldarius Strain Tc-4-1. I Bacteriol 2011; 193:5602-5603.

PubMed doi:10.1128/]B.05709-11

79. Delorme C, Bartholini C, Luraschi M, Pons N, Loux V, Almeida M, Guedon E, Gibrat JF, Renault P. Complete Genome Sequence of the Pigmented Streptococcus thermophilus Strain JIM8232. J Bacteriol 2011; 193:5581-5582. PubMed doi:10.1128/JB.05404-11

80. Ma Z, Geng J, Zhang H, Yu H, Yi L, Lei M. Lu Cping, Fan H-jie, Hu S: Complete Genome Sequence of Streptococcus equi subsp. zooepidemicus Strain ATCC 35246. I Bacteriol 2011; 193:5583-5584. PubMed doi:10.1128/|B.05700$\underline{11}$

81. Yang H, Liao Y, Wang B, Lin Y, Pan L. Complete Genome Sequence of Bacillus amyloliquefaciens $\mathrm{XH7}$, Which Exhibits Production of Purine Nucleosides. J Bacteriol 2011; 193:5593-5594. PubMed doi:10.1128/JB.05880-11

82. Lee $\mathrm{SH}$, Jung JY, Lee $\mathrm{SH}$, Jeon CO. Complete Genome Sequence of Leuconostoc kimchii Strain C2, Isolated from Kimchi. J Bacteriol 2011; 193:5548. PubMed doi:10.1128/JB.05707-11
83. Kim DW, Choi SH, Kang A, Nam SH, Kim DS, Kim RN, Kim A, Park HS. Draft Genome Sequence of Lactobacillus malefermentans KCTC 3548. I Bacteriol 2011; 193:5537. PubMed doi:10.1128/JB.05710-11

84. Lee $\mathrm{SH}$, Jung JY, Lee $\mathrm{SH}$, Jeon CO. Complete Genome Sequence of Weissella koreensis KACC 15510, Isolated from Kimchi. J Bacteriol 2011; 193:5534. PubMed doi:10.1128//B.05704-11

85. Li Y, Zheng $\mathrm{H}$, Liu $\mathrm{Y}$, Jiang $\mathrm{Y}$. Jiuquing $\mathrm{Xi}$, Chen W, Song Z: The Complete Genome Sequence of Mycoplasma bovis Strain Hubei-1. PLoS ONE 2011; 6:e20999.

PubMed doi:10.1371/journal.pone.0020999

86. Shu HW, Liu TT, Chan HI, Liu YM, Wu KM, Shu HY, Tsai SF, Hsiao KJ, Hu WS, Ng WV. Genome Sequence of the Repetitive-Sequence-Rich Mycoplasma fermentans Strain M64. I Bacteriol 2011; 193:4302-4303. PubMed doi:10.1128//B.05228$\underline{11}$

87. Antunes A, Alam I, El Dorry H, Siam R, Robertson A, Bajic VB, Stingl U. Genome Sequence of Haloplasma contractile, an Unusual Contractile Bacterium from a Deep-Sea Anoxic Brine Lake. I Bacteriol 2011; 193:4551-4552.

PubMed doi:10.1128//B.05461-11

88. Yang F, Tang C, Wang Y, Zhang H, Yue H. Genome Sequence of Mycoplasma ovipneumoniae Strain SC01. I Bacteriol 2011; 193:5018. PubMed doi:10.1128/JB.05363-11

89. Kim WJ, Kim YO, Kim DS, Choi SH, Kim DW, Lee JS, Kong HJ, Nam BH, Kim BS, Lee SJ, et al. Draft Genome Sequence of Kocuria rhizophila P7-4. J Bacteriol 2011;

90. Seipke RF, Crossman L, Drou N, Heavens D, Bibb MJ, Caccamo M, Hutchings MI. Draft Genome Sequence of Streptomyces S4, a symbiont of the leafcutter ant Acromyrmex octospinosus. J Bacteriol 2011;

91. Shin NR, Whon TW, Roh SW, Kim MS, Jung MJ, Lee J, Bae JW. Genome Sequence of Corynebacterium nuruki S6-4T, Isolated from Alcohol Fermentation Starter . J Bacteriol 2011;

92. Butler-Wu SM, Sengupta DJ, Kittichotirat W, Matsen FA, Bumgarner RE. Genome Sequence of a Novel Species, Propionibacterium humerusii. J Bacteriol 2011; 193:3678. PubMed doi:10.1128//B.05036-11

93. Zhang ZY, Sun ZQ, Wang ZL, Wen ZL, Sun QW, Zhu ZQ, Song YZ, Zhao JW, Wang HH, Zhang SL, et al. Complete Genome Sequence of a Novel 
Clinical Isolate, the Nontuberculous Mycobacterium Strain JDM601. J Bacteriol 2011; 193:43004301. PubMed doi:10.1128/JB.05291-11

94. Erxleben A, Wunsch-Palasis J, Gruning BA, Luzhetska M, Bechthold A, Gunther S. Genome Sequence of Streptomyces sp. Strain Tu6071. J Bacteriol 2011; 193:4278-4279.

PubMed doi:10.1128/JB.00377-11

95. O'Connell Motherway M, Zomer A, Leahy SC, Reunanen J, Bottacini F, Claesson MJ, O'Brien F, Flynn K, Casey PG, Moreno Munoz JA, et al. Functional genome analysis of Bifidobacterium breve UCC2003 reveals type IVb tight adherence (Tad) pili as an essential and conserved hostcolonization factor. Proc Natl Acad Sci USA 2011; 108:11217-11222. $\underline{\text { PubMed doi:10.1073/pnas.1105380108 }}$

96. Hunyadkurti J, Feltoti Z, Horvath B, Nagymihaly M, Voros A, McDowell A, Patrick S, Urban E, Nagy I. Complete Genome Sequence of Propionibacterium acnes Type IB strain 6609. J Bacteriol 2011; 193: doi:10.1128/JB.05372-11

97. Cai M, Chen WM, Nie Y, Chi CQ, Wang YN, Tang YQ, Li GY, Wu XL. Complete Genome Sequence of Amycolicicoccus subflavus DQS39A1T, an Actinomycete Isolated from Crude OilPolluted Soil. J Bacteriol 2011; 193:4538-4539. PubMed doi:10.1128/JB.05388-11

98. Ge F, Li W, Chen G, Liu Y, Zhang G, Yong B, Wang Q, Wang N, Huang Z, Li W, et al. Draft Genome Sequence of Gordonia neofelifaecis NRRL B-59395, a Cholesterol-Degrading Actinomycete. / Bacteriol 2011; 193:5045-5046. PubMed doi:10.1128/JB.05531-11

99. Sales CM, Mahendra S, Grostern A, Parales RE, Goodwin LA, Woyke T, Nolan M, Lapidus A, Chertkov O, Ovchinnikova G, et al. Genome Sequence of the 1,4-Dioxane-Degrading Pseudonocardia dioxanivorans Strain CB1190. J Bacteriol 2011; 193:4549-4550.

PubMed doi:10.1128//B.00415-11

100. Ham JS, Lee T, Byun MJ, Lee KT, Kim MK, Han GS, Jeong SG, Oh MH, Kim DH, Kim H. Complete Genome Sequence of Bifidobacterium longum subsp. longum KACC 91563. J Bacteriol 2011; 193:5044. PubMed doi:10.1128/JB.05620$\underline{11}$

101. Barbe V, Bouzon M, Mangenot S, Badet B, Poulain J, Segurens B, Vallenet D, Marliere P, Weissenbach J. Complete Genome Sequence of Streptomyces cattleya NRRL 8057, a Producer of Antibiotics and Fluorometabolites. J Bacteriol 2011;
193:5055-5056. PubMed doi:10.1128/JB.0558311

102. Yang X, Xue R, Shen C, Li S, Gao C, Wang Q, Zhao X. Genome Sequence of Rhodococcus sp. Strain R04, a Polychlorinated-Biphenyl Biodegrader. J Bacteriol 2011; 193:5032-5033. PubMed doi:10.1128//B.05635-11

103. Gomes LHF, Otto TD, Vasconcellos EA, Ferrao PM, Maia RM, Moreira AS, Ferreira MA, CastelloBranco LRR, Degrave WM, Mendonca-Lima L. Genome Sequence of Mycobacterium bovis BCG Moreau, the Brazilian Vaccine Strain against Tuberculosis. J Bacteriol 2011; 193:5600-5601.

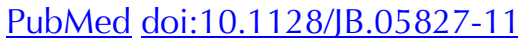

104. Pan Y, Yang X, Li J, Zhang R, Hu Y, Zhou Y, Wang J, Zhu B. Genome Sequence of the Spinosyns-Producing Bacterium Saccharopolyspora spinosa NRRL 18395. J Bacteriol 2011; 193:31503151. PubMed doi:10.1128/JB.00344-11

105. Zhang Y, Chen C, Liu J, Deng H, Pan A, Zhang L, Zhao X, Huang M, Lu B, Dong $\mathrm{H}$, et al. Complete Genome Sequences of Mycobacterium tuberculosis Strains CCDC5079 and CCDC5080, Which Belong to the Beijing Family. J Bacteriol 2011; 193:5591-5592. PubMed doi:10.1128/JB.0545211

106. Verma M, Kaur J, Kumar M, Kumari K, Saxena A, Anand S, Nigam A, Ravi V, Raghuvanshi S, Khurana $\mathrm{P}$, et al. Whole Genome Sequence of the $\mathrm{Ri}$ famycin B-Producing Strain Amycolatopsis mediterranei S699. J Bacteriol 2011; 193:5562-5563. PubMed doi:10.1128/JB.05819-11

107. Sarikhan S, Azarbaijani R, Yeganeh LP, Fazeli AS, Amoozegar MA, Salekdeh GH. Draft Genome Sequence of Nesterenkonia sp. Strain F, Isolated From Aran-Bidgol Salt Lake in Iran. J Bacteriol 2011; 193:5580. PubMed doi:10.1128/JB.05808$\underline{11}$

108. Zhao X, Yang T. Draft Genome Sequence of the Marine Sediment-Derived Actinomycete Streptomyces xinghaiensis NRRL B24674T. I Bacteriol 2011; 193:5543. PubMed doi:10.1128//B.0568911

109. Sait M, Clark EM, Wheelhouse N, Livingstone $M$, Spalding L, Siarkou VI, Vretou E, Smith DGE, Lainson FA, Longbottom D. Genome sequence of the Chlamydophila abortus variant strain LLG. . J Bacteriol 2011; 193:4276-4277. PubMed doi:10.1128/JB.05290-11

110. Grinblat-Huse V, Drabek EF, Creasy HH, Daugherty SC, Jones KM, Santana-Cruz I, Tallon LJ, Read TD, Hatch TP, Bavoil P, et al. Genome Se- 
quences of the Zoonotic Pathogens Chlamydia

psittaci 6BC and Cal10. J Bacteriol 2011;

193:4039-4040. PubMed doi:10.1128/JB.0527711

111. Somboonna N, Wan R, Ojcius DM, Pettengill MA, Joseph SJ, Chang A, Hsu R, Read TD, Dean D: Hypervirulent Chlamydia trachomatis Clinical Strain Is a Recombinant between Lymphogranuloma Venereum (L2) and D Lineages . mBio May, 2.

112. Angelov A, Loderer C, Pompei S, Liebl W. A novel family of carbohydrate-binding modules revealed by the genome sequence of Spirochaeta thermophila DSM 6192. App/ Environ Microbiol 2011; 77:5483-5489. PubMed doi:10.1128/AEM.00523-11

113. Håfström T, Jansson D, Segerman B. Complete Genome Sequence of Brachyspira intermedia Reveals Unique Genomic Features in Brachyspira Species and Phage-mediated Horizontal Gene Transfer. BMC Genomics 2011; 12:395. PubMed doi:10.1186/1471-2164-12-395

114. Watanabe T, Maruyama F, Nozawa T, Aoki A, Okano S, Shibata Y, Oshima K, Kurokawa K, Hattori M, Nakagawa I, et al. Complete Genome Sequence of the Bacterium Porphyromonas gingivalis TDC60, Which Causes Periodontal Disease. J

Bacteriol 2011; 193:4259-4260.

PubMed doi:10.1128/JB.05269-11

115. Klippel B, Lochner A, Bruce DC, Walston Davenport K, Detter C, Goodwin LA, Han J, Han S, Hauser L, Land ML, et al. Complete Genome Sequences of Krokinobacter sp. Strain 4H-3-7-5 and Lacinutrix sp. Strain 5H-3-7-4, PolysaccharideDegrading Members of the Family Flavobacteriaceae. J Bacteriol 2011; 193:4545-4546. PubMed doi:10.1128/JB.05518-11

116. Du Z, Zhang Z, Miao T, Wu J, Lu G, Yu J, Xiao J, Chen G. Draft Genome Sequence of the Novel Agar-Digesting Marine Bacterium HQM9. J Bacteriol 2011; 193:4557-4558.

PubMed doi:10.1128/JB.05513-11

117. Gao Z, Liu X, Ruan L. Genome Sequence of Anaerophaga sp. Strain HS1, a Novel, Moderately Thermophilic, Strictly Anaerobic Bacterium Isolated from Hot Spring Sediment. J Bacteriol 2011; 193:5572. PubMed doi:10.1128/JB.05719-11

118. Manfredi P, Pagni M, Cornelis GR. Complete Genome Sequence of the Dog Commensal and Human Pathogen Capnocytophaga canimorsus Strain 5. J Bacteriol 2011; 193:5558-5559. PubMed doi:10.1128/JB.05853-11
119. Oh C, Heo SJ, De Zoysa M, Affan A, Jung WK, Park HS, Lee Y, Lee J, Yoon KT, Kang DH. Whole-Genome Sequence of the XylanaseProducing Mesoflavibacter zeaxanthinifaciens Strain S86. J Bacteriol 2011; 193:5557. PubMed doi:10.1128/JB.05793-11

120. Swithers KS, DiPippo JL, Bruce DC, Detter C, Tapia R, Han S, Goodwin LA, Han J, Woyke T, Pitluck S, et al. Genome Sequence of Kosmotoga olearia Strain TBF 19.5.1, a Thermophilic Bacterium with a Wide Growth Temperature Range, Isolated from the Troll B Oil Platform in the North Sea. J Bacteriol 2011; 193:5566-5567. PubMed doi:10.1128/JB.05828-11

121. Kim BK, Jung MY, Yu DS, Park SJ, Oh TK, Rhee SK, Kim JF. Genome Sequence of an AmmoniaOxidizing Soil Archaeon, "Candidatus Nitrosoarchaeum koreensis" MY1. I Bacteriol 2011; 193:5539-5540. PubMed doi:10.1128/JB.05717$\underline{11}$

122. Wóycicki R, Witkowicz J, Gawroński P, Dąbrowska J, Lomsadze A, Pawełkowicz M, Siedlecka E, Yagi K, Pląder W, Seroczyńska A, et al. The Genome Sequence of the North-European Cucumber (Cucumis sativus L.) Unravels Evolutionary Adaptation Mechanisms in Plants. PLoS ONE 2011; 6:e22728. $\underline{\text { PubMed doi:10.1371/journal.pone.0022728 }}$

123. Rivarola M, Foster JT, Chan AP, Williams AL, Rice DW, Liu X, Melake-Berhan A, Huot Creasy H, Puiu D, Rosovitz MJ, et al. Castor Bean Organelle Genome Sequencing and Worldwide Genetic Diversity Analysis. PLOS ONE 2011; 6:e21743. PubMed doi:10.1371/journal.pone.0021743

124. Belaganahalli MN, Maan S, Maan NS, Tesh R, Attoui H, Mertens PPC. Umatilla Virus Genome Sequencing and Phylogenetic Analysis: Identification of Stretch Lagoon Orbivirus as a New Member of the Umatilla virus Species. PLOS ONE 2011; 6:e23605.

$\underline{\text { PubMed doi:10.1371/journal.pone.0023605 }}$

125. Star B, Nederbragt AJ, Jentoft S, Grimholt U, Malmstrom M, Gregers TF, Rounge TB, Paulsen J, Solbakken $\mathrm{MH}$, Sharma A, et al.: The genome sequence of Atlantic cod reveals a unique immune system . Nature 2011, advance online publication.

126. Genome sequence and analysis of the tuber crop potato. Nature 2011; 475:189-195. PubMed doi:10.1038/nature10158

127. Zsak L, Day J, Oakley B, Seal B: The complete genome sequence and genetic analysis of ФCA82 
a novel uncultured microphage from the turkey gastrointestinal system. [date unknown], 8:331.

128. Barth D, Berendonk T. The mitochondrial genome sequence of the ciliate Paramecium caudatum reveals a shift in nucleotide composition and codon usage within the genus Paramecium. BMC Genomics 2011; 12:272.

PubMed doi:10.1186/1471-2164-12-272

129. Jiang $X$, Jiang $H$, Li C, Wang S, Mi Z, An X, Chen J, Tong Y: Sequence characteristics of T4like bacteriophage IME08 genome termini revealed by high throughput sequencing. [date unknown], 8:194.

130. Lee SW, Markham P, Markham J, Petermann I, Noormohammadi A, Browning G, Ficorilli N, Hartley C, Devlin J. First complete genome sequence of infectious laryngotracheitis virus. BMC Genomics 2011; 12:197.

PubMed doi:10.1186/1471-2164-12-197
131. Renfree MB, Papenfuss AT, Deakin JE, Lindsay J, Heider T, Belov K, Rens W, Waters PD, Pharo EA, Shaw G, et al. Genome sequence of an Australian kangaroo, Macropus eugenii, provides insight into the evolution of mammalian reproduction and development. Genome Biol 2011; 12:R81. PubMed doi:10.1186/gb-2011-12-8-r81

132. Han X, Zhang W, Xue Y, Shao S. Sequence analysis reveals mosaic genome of Aichi virus. Virol J 2011 ; 8:390. PubMed doi:10.1186/1743-422X-8$\underline{390}$

133. López-Madrigal S, Latorre A, Porcar M, Moya A, Gil R. Complete Genome Sequence of "Candidatus Tremblaya princeps" Strain PCVAL, an Intriguing Translational Machine below the LivingCell Status. J Bacteriol 2011; 193:5587-5588. PubMed doi:10.1128/JB.05749-11 